\title{
Science is social
}

\author{
Well-designed science education via social media may help to reach a larger group of audiences with the aim \\ of reducing the boundaries between researchers and the public. This may, in turn, move basic science toward \\ translational improvement in human health and agriculture.
}

A high replication rate is a great thing to have if one is a norovirus with a one-click trick to make people sick and agitated enough to pass it on. Social media is similarly prone to endless repetition of ugly nonsense, as what transmits best virally is a sense of discomfort just novel or nasty enough to ensure transmission. Humans, who need to stick around a bit longer, had better interact with give and take, exchange and prioritize useful information, learn and accommodate.

Many web users are not in fact social in any respect, and their tweeting and posting are skewed toward announcing, advertising and broadcasting, as if they were still operating in traditional media like cave painting, bumper stickers, billboards, radio or television. This abuse of new tools for old ends seems not to realize the full potential of the technology. To reclaim the promise of the World Wide Web to inform, engage and enlighten us all, we scientists should consider how to add the missing social elements of content, purpose and interaction.

As examples, we can explore two use cases in social media spreading the "Share this article" links found at the end of every manuscript webpage giving access to the full content of our research papers. First, when educators send reading assignments to their students via Twitter or WeChat, we envision a possible competition for ways to assess their reading comprehension most elegantly and efficiently within a minimally modified mobile browser or app. Students might perform analysis of a single publication to identify the main claims, evidence and method, and the logical dependencies of each of these upon the preceding claim (Nat. Genet. 47, 305, 2015). Another assignment could be synthesis, extracting supporting or contrasting claims from multiple publications using different approaches to propose their own hypotheses, predictions and experiments. The second case is to answer the questions of non-scientists by semantic matching of lay questions with researchers and the publications they know (Nat. Genet. 45, 1411, 2013). As such, it's expected that science education and communication via social media could be efficient and participatory if well designed by scientists, editors and authors.

Another advantage we can take from social media is for public understanding of science, for the journal to reach a larger group of audiences with the aim of facilitating a true exchange of information and understanding between researchers and the public. The brief format of many social media outlets seems poised to provide easy access to science content, concentrated to a simple link, a title and a brief summary. We should try to facilitate the interest and participation of specific audience groups who have a vested interest in scientific questions relevant to their real life. For example, patients who suffer from common diseases or farmers and breeders who are keen in agricultural improvement would appreciate the valuable information from genomic studies for human disease or agricultural traits in crops; they may also be interested in sharing these scientific insights and current events with their families and communities. Social media should be appreciated for bringing new science to our daily life in a more friendly and efficient way, without requirements of in-depth reading for research papers, with the aim of better communications between authors and readers and between scientists and the public.

Currently, social media dynamics are incentivized by quantification of the rapidity of viral spread and the amount of attention hijacked. However, a hopeful feature has emerged in these media because of their interactive nature. Trust and reputation are established among users by returning favors such as publicizing or searching for information via one's contacts. So, maybe we also need measurements of the accuracy and quality of social engagement and interaction.

The measure of better science is to build a better life in human society via optimization and integration of science contents and social elements, with efforts from all of us.

Published online: 28 November 2018 https://doi.org/10.1038/s41588-018-0308-4 